\title{
Erratum to "Non Self Adjoint Operators, Infinite Determinants, and Some Applications," Russ. J. Math. Phys. 12, 443-471 (2005)
}

\author{
F. Gesztesy, ${ }^{*, 1}$ Y. Latushkin, ${ }^{* *, 2}$ M. Mitrea ${ }^{*, 3}$ and M. Zinchenko ${ }^{* * *, 4}$ \\ *Department of Mathematics, Baylor University, One Bear Place \#97328, Waco, TX 76798-7328, USA \\ ** Department of Mathematics, University of Missouri, Columbia, MO 65211, USA \\ *** Department of Mathematics and Statistics, University of New Mexico, Albuquerque, NM 87131, USA, \\ E-mail: ${ }^{1}$ Fritz_Gesztesy@baylor .edu, ${ }^{2}$ latushkiny@missouri.edu, \\ ${ }^{3}$ Marius_Mitrea@baylor .edu, ${ }^{4}$ maxim@math.unm.edu
}

Received May 12, 2020; Revised May 13, 2020; Accepted May 20, 2020

DOI 10.1134/S1061920820030127

The following corrections should be made in [2]:

The additional hypothesis: each connected component of $\mathbb{C} \backslash \widetilde{\sigma}_{\mathrm{e}}\left(H_{0}\right)$ contains a point of $\rho(H)$, and each connected component of $\mathbb{C} \backslash \widetilde{\sigma}_{\mathrm{e}}(H)$ contains a point of $\rho\left(H_{0}\right)$, should be made in Theorem 4.5, Remark 4.6, Theorem 5.4, and Remark 5.5.

For a detailed discussion of this circle of ideas see [1, Section 6].

\section{REFERENCES}

[1] J. Behrndt, A. F. M. ter Elst, and F. Gesztesy, The Generalized Birman-Schwinger Principle, arXiv 2005.01195

[2] F. Gesztesy, Y. Latushkin, M. Mitrea, and M. Zinchenko, "Nonselfadjoint Operators, Infinite Determinants, and Some Applications," Russ. J. Math. Phys. 12, 443-471 (2005). For a corrected and considerably updated version of sections 4 and 5 of this paper, see the latest version of arXiv math/0511371 